\title{
Radiative Heat Loss Correction for 3-Body Graphite
} Calorimeters

\author{
C. $\operatorname{IONITA}^{a}$, D. RADU ${ }^{b}$ AND I. ASTEFANOAEI ${ }^{b}$ \\ ${ }^{a}$ S.C. Explora Rx S.R.L., Computer Tomography Clinic, Str. Eternitate, 45, 700304, Iasi, Romania \\ ${ }^{b}$ Department of Solid State and Theoretical Physics, Faculty of Physics \\ Univ. "Al.I. Cuza", Blvd. Carol I, 11, 700506, Iasi, Romania
}

(Received May 20, 2009; in final form June 2, 2010)

For a 3-body Domen-type absorbed-dose graphite calorimeter a refined ordinary differential equation model is developed taking into account the heat loss through radiation from the surface of all three bodies. The new reduced heat transfer coefficients, $K_{i}^{*}, i=1,2,3$ were defined and the associated calculated heat loss correction factors $F_{\mathrm{C}}^{*}$ and $F_{\mathrm{C}+\mathrm{J}}^{*}$ were determined. The total energy lost by radiation from the core surface was evaluated for both heating and post heating periods as being 31.35\% from the total core heat loss (and up to 1.1\% from the total energy delivered to the core during the heating time). A new correction factor for heat loss through radiation from the calorimeter bodies' surfaces was introduced and calculated as being $k_{\mathrm{SB}} \approx 0.9996$.

PACS numbers: 29.40.Vj, 44.40.+a

\section{Introduction}

The design of graphite calorimeters depends on knowledge of the mechanisms of heat transfer, the relation between changes in heat and temperature, and the methods of temperature measurement and control.

As it is well known, there are three distinct phenomena connected with heat transfer: convection, conduction and radiation. Actual primary standard graphite calorimeters usually work at air pressures below $0.001 \mathrm{~Pa}$. At such pressure values only radiative processes can contribute to heat transfer through air. Indeed, as it is known, three correlated quantities are important for establishing if the natural convection can occur in air or not: the air pressure, $P$, the temperature difference between the two surfaces, $\Delta T$, and the characteristic distance (the separation) between the two surfaces, $d$. Under normal atmospheric pressure conditions, the dominant mode of heat transfer through air is usually convection. The word usually was used to indicate that in actual graphite calorimeters significant convection does not occur because of small gaps ( $1 \mathrm{~mm}$ or smaller) and small temperature rises $\left(\approx 10^{-3} \mathrm{~K}\right)^{*}$. In the process of thermal convection, heat is transported by air movement. Below the pressure value of about $0.1 \mathrm{~Pa}\left(\right.$ i.e. $\approx 10^{-3} \mathrm{~mm} \mathrm{Hg}$ ) the fluid model of the air breaks down (because the molecular mean free path of air becomes greater than $d$ ) and the inequality that correlates the three quantities mentioned above is satisfied by a factor of $10^{11}$ [1]. Since the usual air pressure in a graphite calorimeter varies in the interval $10^{-4} \div 10^{-3} \mathrm{~Pa}$, it comes that in this case the natural convection is physically quasi-impossible.

\footnotetext{
* It can be shown that natural convection will not occur at atmospheric pressure provided that $\Delta T$ is less than $1.1 \mathrm{~K}[1]$.
}

Thus, in most of graphite calorimeters only conduction and radiation mechanisms can contribute to heat transfer through air. The comparative importance of heat transfer by conduction through the residual air and by thermal radiation depends on the vacuum pressure and on the surface and geometric conditions of the bodies (the core, the jacket, the shield and the medium).

The phenomenon of conduction through gases is discussed by numerous authors (e.g. [2-4]). If the mean free path of the air molecules is smaller than the spacing between the bodies, the heat transfer takes place almost entirely by conduction through air. Obviously, the mean free path of the air molecules, $\lambda$, depends essentially on the pressure value. For instance, at near $7 \mathrm{~Pa}$ (i.e. $0.05 \mathrm{~mm} \mathrm{Hg}) \lambda \approx 1 \mathrm{~mm}$ (this value for air pressure was chosen because it leads to a mean free path that is comparable with the usual distances between the bodies of a graphite calorimeter). This means that for a spacing between the bodies that has a dimension comparable with $1 \mathrm{~mm}$, if the pressure is greater than $7 \mathrm{~Pa}$ then the heat transfer is almost entirely by conduction through air and it is quasi-constant. For pressure values below $7 \mathrm{~Pa}$ (which represents a transitional region in this situation) the conduction through air begins to decrease. Further decreases of pressure will produce a corresponding linear decrease of heat conduction. This decrease continues until the rate of heat transfer by conduction equals the rate of heat transfer by radiation. For a thermal emissivity value $\varepsilon=0.1$ this happens when the pressure is reduced to about $0.5 \mathrm{~Pa}(\approx 0.004 \mathrm{~mm} \mathrm{Hg})$. This represents the second transitional point because below this pressure value (assuming that all other parameters are unchanged) the conduction heat transfer becomes more and more negligible and the dominant heat transfer phenomenon is the radiation. The schematic representation of the connection between the air pressure value and the dominant heat transfer process is given in Fig. 1. 


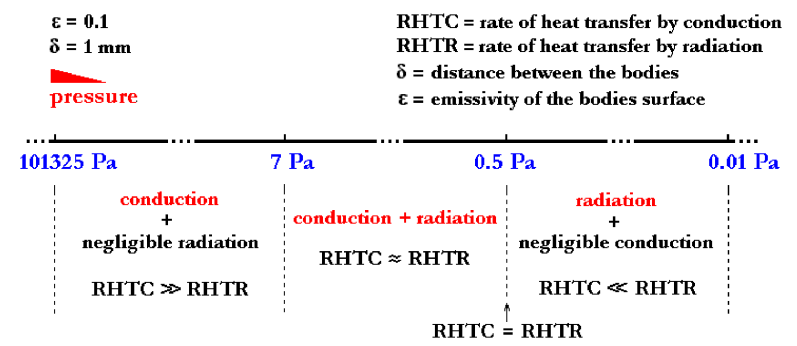

Fig. 1. Schematic representation of the connection between the air pressure value and the dominant heat transfer process.

Taking into account that the usual working values of the pressure in the graphite calorimeter existing at ENEA - Istituto Nazionale di Metrologia delle Radiazioni Ionizzanti, Centro Ricerche Casaccia (Roma, Italy) are about $0.006 \mathrm{~Pa}$, it can be concluded that from the three possible heat transfer mechanisms through air, only the radiation remains as significant.

In order to check up if the contribution of the radiative heat loss is indeed significant or if it can be neglected within the measurement uncertainty of the actual graphite calorimeter operating at ENEA-INMRI, a theoretical model based on differential heat conduction equation will be used. Some simplified models of the temperature exchange have been already presented by Domen and Lamperti [5] and Janssens et al. [6] in the case of quasi-adiabatic mode of operation.

Before starting the analysis, a few comments on some basic difficulties of such a theoretical model (that have been put into evidence especially by Picard et al. [7, 8]) are worthwhile to be done. As Dr. Picard pointed out, there are - at least - two major difficulties that arise, namely:

1. an approach using the heat equation is appropriate provided that a very stable environment is present; in fact/usually the laboratory temperature is not so stable;

2. the heat flow via radiation loss is present, which will add a term proportional to $T^{4}$.

The second observation is actually related to the main aim of the present report: to analyze the effect of radiative processes on thermal behaviour of a 3-body graphite calorimeter. This means that radiative heat loss must be taken into account. From the mathematical point of view, this fact leads to a differential equation system that is no longer linear, nor homogeneous. In order to obtain a unique solution, the resulting Riccati-type equations must be solved. Since an analytical solution of such equation system is quasi-impossible to find out, the use of a numerical approach is unavoidable.

The first difficulty signalized by Picard is more important and must carefully be analyzed for each particular case. In this view, it is important to observe that in this report $T_{1}, T_{2}$ and $T_{3}$ do not represent the temperature differences with respect to laboratory of the graphite cylinder, vacuum chamber and laboratory environment — as in the report of Picard (see Fig. 16 in [7]), but they represent the temperature rises $(\mathrm{K})$ of the core, jacket and shield, respectively, above the constant temperature of the surrounding medium ${ }^{\dagger}$. Besides, if the graphite calorimeter works in the quasi-isothermal mode of operation (or constant-temperature operating mode - as it is named by Daures and Ostrowsky [9] from CEA Saclay, DETECS - LNHB, France), then the condition in the first observation becomes less important. Indeed, the rapid temperature changes that prevent direct measurement of the temperature differences that determine heat gain would be eliminated by operating the calorimeter in quasi-isothermal mode [10].

Although the present considerations refer only to the heat loss-compensated mode of operation [11] (when heat is applied only to the core: $\left.P_{1} \neq 0, P_{2}=P_{3}=0\right)$, the base model which takes into account the radiative heat loss correction (RHLC) remains also valid for quasi-adiabatic and quasi-isothermal modes of operation.

\section{The RHLC model}

As it was also proposed by Picard et al. [8] from BIPM (France), in order to find the influence on temperature changes brought by the radiative heat loss, instead the problem (1) from paper of Janssens et al. [6] (and also - in an older version - from the paper of Domen and Lamperti [5]) the following mathematical problem must be solved:

$$
(C)(\dot{T})=(P)-(K)(T)-(e)\left(T_{\mathrm{rad}}^{4}\right)+\left(e_{3}\right)\left(T_{\mathrm{M}}^{4}\right),
$$

where, in the 3-body calorimeter model,

$$
\begin{aligned}
& (C)=\left(\begin{array}{ccc}
C_{1} & 0 & 0 \\
0 & C_{2} & 0 \\
0 & 0 & C_{3}
\end{array}\right), \\
& (T)=\left(\begin{array}{c}
T_{1} \\
T_{2} \\
T_{3}
\end{array}\right), \quad(\dot{T})=\left(\begin{array}{c}
\dot{T}_{1} \\
\dot{T}_{2} \\
\dot{T}_{3}
\end{array}\right)=\left(\begin{array}{c}
\frac{\mathrm{d} T_{1}}{\mathrm{~d} t} \\
\frac{\mathrm{d} T_{2}}{\mathrm{~d} t} \\
\frac{\mathrm{d} T_{3}}{\mathrm{~d} t}
\end{array}\right), \\
& \left(T_{\mathrm{rad}}^{4}\right)=\left(\begin{array}{c}
\left(T_{\mathrm{M}}+T_{1}\right)^{4} \\
\left(T_{\mathrm{M}}+T_{2}\right)^{4} \\
\left(T_{\mathrm{M}}+T_{3}\right)^{4}
\end{array}\right), \quad\left(T_{\mathrm{M}}^{4}\right)=\left(\begin{array}{c}
0 \\
0 \\
T_{\mathrm{M}}^{4}
\end{array}\right) \text {, } \\
& (P)=\left(\begin{array}{c}
P_{1} \\
P_{2} \\
P_{3}
\end{array}\right) \text {, } \\
& (K)=\left(\begin{array}{ccc}
K_{1} & -K_{1} & 0 \\
-K_{1} & K_{1}+K_{2} & -K_{2} \\
0 & -K_{2} & K_{2}+K_{3}
\end{array}\right),
\end{aligned}
$$

and

\footnotetext{
$\dagger$ The temperature of the medium is kept quasi-constant through a PID controller.
} 


$$
\begin{aligned}
& (e)=\left(\begin{array}{ccc}
e_{1} & -e_{1} & 0 \\
-e_{1} & e_{1}+e_{2} & -e_{2} \\
0 & -e_{2} & e_{2}+e_{3}
\end{array}\right), \\
& \left(e_{3}\right)=\left(\begin{array}{lll}
0 & 0 & 0 \\
0 & 0 & 0 \\
0 & 0 & e_{3}
\end{array}\right) .
\end{aligned}
$$

In the above relations $T_{\mathrm{M}}=T_{0}+T_{\text {medium }}\left[{ }^{\circ} \mathrm{C}\right]=$ $273.15+25=298.15 \mathrm{~K}$ represents the constant medium temperature in kelvins. The significance and the methods used to find the expressions and/or the numerical values for $(C),(P)$ and $(T)$ in (1) were explained in detail in $[5,6]$. For this reason here only the meaning of the matrices $(K)$ and $(e)$ will be given ${ }^{\ddagger}$. In Eq. (1), (e) represents a coefficient matrix combining the emissivity and geometrical factors of the bodies in a certain experimental arrangement (in the present case, the 3-body graphite calorimeter). The explicit expressions of the matrix elements $e_{i}, i=1,2,3$ can be found using both the StefanBoltzmann law and the McAdams [12] approximation for the net rate of radiative heat transfer between a suspended body and its enclosure. Because the geometric structure of a Domen-type graphite calorimeter [5] consists of a succession of nested bodies (the medium is in fact the enclosure for the shield; the shield is the enclosure for the jacket, which in its turn is the enclosure for the core - see Fig. 2) this approximation proves to be very good. Thus, the explicit form of the equation system (1) becomes

$$
\left\{\begin{array}{l}
C_{1} \dot{T}_{1}=P_{1}-K_{1}\left(T_{1}-T_{2}\right) \\
\quad-e_{1}\left[\left(T_{\mathrm{M}}+T_{1}\right)^{4}-\left(T_{\mathrm{M}}+T_{2}\right)^{4}\right] \\
C_{2} \dot{T}_{2}=P_{2}+K_{1}\left(T_{1}-T_{2}\right)-K_{2}\left(T_{2}-T_{3}\right) \\
\quad+e_{1}\left[\left(T_{\mathrm{M}}+T_{1}\right)^{4}-\left(T_{\mathrm{M}}+T_{2}\right)^{4}\right] \\
\quad-e_{2}\left[\left(T_{\mathrm{M}}+T_{2}\right)^{4}-\left(T_{\mathrm{M}}+T_{3}\right)^{4}\right] \\
C_{3} \dot{T}_{3}=P_{3}+K_{2}\left(T_{2}-T_{3}\right)-K_{3} T_{3} \\
\quad+e_{2}\left[\left(T_{\mathrm{M}}+T_{2}\right)^{4}-\left(T_{\mathrm{M}}+T_{3}\right)^{4}\right] \\
\quad-e_{3}\left[\left(T_{\mathrm{M}}+T_{3}\right)^{4}-T_{\mathrm{M}}^{4}\right]
\end{array}\right.
$$

where

$$
\begin{aligned}
e_{1} & \equiv e_{\mathrm{c}-\mathrm{j}}=\sigma A_{\mathrm{c}} F_{\mathrm{c}-\mathrm{j}} \\
& =\frac{\sigma A_{\mathrm{c}}}{\varepsilon_{\mathrm{g}}^{-1}+\left(A_{\mathrm{c}} / A_{\mathrm{j}}^{\mathrm{int}}\right)\left(\varepsilon_{\mathrm{My}}^{-1}-1\right)}, \\
e_{2} & \equiv e_{\mathrm{j}-\mathrm{sh}}=\sigma A_{\mathrm{j}}^{\mathrm{ext}} F_{\mathrm{j}-\mathrm{sh}}
\end{aligned}
$$

\footnotetext{
$\ddagger$ The appearance of the constant matrices $\left(e_{3}\right)$ and $\left(T_{\mathrm{M}}^{4}\right)$ in the matrix Eq. (1) is due - on the one hand - to the fact that in the Stefan-Boltzmann law, the absolute temperature of the corresponding surfaces appear and - on the other hand - to the nonlinearity in temperature of the same law.
}

$$
=\frac{\sigma A_{\mathrm{j}}^{\text {ext }}}{\varepsilon_{\mathrm{g}}^{-1}+\left(A_{\mathrm{j}}^{\mathrm{ext}} / A_{\mathrm{sh}}^{\mathrm{int}, \mathrm{My}}\right)\left(\varepsilon_{\mathrm{My}}^{-1}-1\right)},
$$

and

$$
\begin{aligned}
e_{3} & \equiv e_{\mathrm{sh}-\mathrm{m}}=\sigma A_{\mathrm{sh}}^{\mathrm{ext}, \mathrm{My}} F_{\mathrm{sh}-\mathrm{m}} \\
& =\frac{\sigma A_{\mathrm{sh}}^{\mathrm{ext}, \mathrm{My}}}{\varepsilon_{\mathrm{My}}^{-1}+\left(A_{\mathrm{sh}}^{\mathrm{ext}, \mathrm{My}} / A_{\mathrm{m}}^{\mathrm{int}, \mathrm{My}}\right)\left(\varepsilon_{\mathrm{My}}^{-1}-1\right)} .
\end{aligned}
$$

In relations (7) the subscript letter indices "c", "j", "sh", "m", "g" and "My" stand for "core", "jacket", "shield", "medium", "graphite" and "aluminized Mylar" respectively, whereas superscript letter indices "int" and "ext" stand for "interior" and "exterior", respectively.
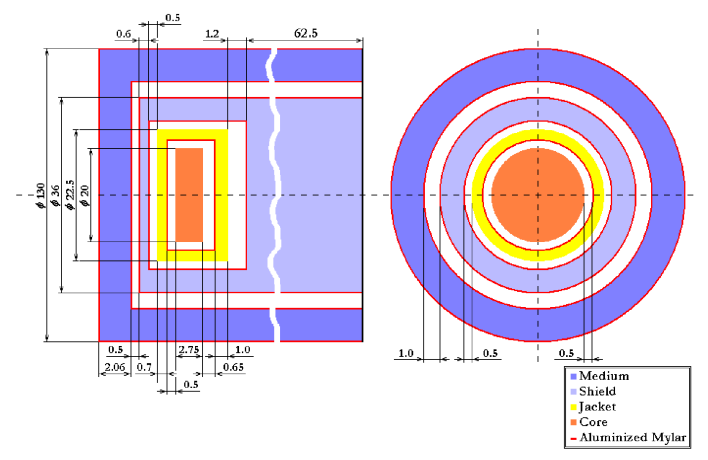

Fig. 2. Schematic configuration of the vacuum gaps in the ENEA-INMRI graphite calorimeter (not at scale). The data in this figure were obtained through the amiability of Dr. A.S. Guerra from ENEA-INMRI, Italy.

Although the influence of the nonlinearity in the equations is virtually undetectable, the authors preferred to use the difference ${ }^{\S}\left(T_{\mathrm{M}}+T_{1}\right)^{4}-\left(T_{\mathrm{M}}+T_{2}\right)^{4}$ instead of linear approximation $4 T_{\mathrm{M}}^{3}\left(T_{1}-T_{2}\right)$ because, firstly, an exact solution is always preferable to an approximate one and, secondly, there is no real difficulty in mathematical handling of this nonlinear term.

Most of the authors use the value of $\varepsilon_{\mathrm{My}} \approx 0.1$ for the emissivity of aluminized Mylar, but, as it was observed by Domen [13]: "a significant error may result when one calculates the heat transfer by radiation using a value of $\varepsilon$ from the literature". Thus, in this report, for the emissivity of aluminized Mylar the value determined by Domen [13] was considered, $\varepsilon_{\mathrm{My}}=0.044$. Concerning the emissivity of graphite, the situation is more or less the same. Although for convenience many authors use "the typical" value of $\varepsilon_{\mathrm{g}} \approx 0.9$, in fact there is a big number of reliable references (see for instance [1, 14, 15]), in which a value closer to 0.8 or less is reported. For this reason, in the present report for the graphite emissivity the value $\varepsilon_{\mathrm{g}}=0.82$ was considered, corresponding to a relatively small absolute temperature interval centred on

\footnotetext{
$\S$ And all similar other.
} 
298 K. Taking all these considerations into account, for the particular geometry of the actual graphite calorimeter existing at ENEA-INMRI (see Fig. 2), the following numerical values of the matrix elements $e_{i}, i=1,2,3$ were found:

$$
\left\{\begin{array}{l}
e_{1}=0.232 \times 10^{-11} \mathrm{~W} \mathrm{~K}^{-4} \\
e_{2}=0.344 \times 10^{-11} \mathrm{~W} \mathrm{~K}^{-4} \\
e_{3}=1.157 \times 10^{-11} \mathrm{~W} \mathrm{~K}^{-4}
\end{array}\right.
$$

For the geometry of the same graphite calorimeter the matrix element $e_{4} \equiv e_{\text {medium-environment }}=5.559 \times$ $10^{-11} \mathrm{~W} \mathrm{~K}^{-4}$ was also evaluated, which can be eventually used to solve the corresponding problem for an improved 4-body calorimeter model. It is worth to specify that all the numerical values of $e_{i}, i=1,2,3,4$ are affected by some small errors due to having neglected the influence of the supports that keep each body in its own fixed place. For instance, the cross-sectional area of the three small polystyrene supports of the core was neglected $\left(A_{\mathrm{c}}^{\text {supports }}=0.589 \times 10^{-6} \mathrm{~m}^{2}\right.$ and it is only $1 / 1360$ of $\left.A_{\mathrm{c}}\right)$. In this model it is assumed that the temperature measured by the sensing thermistors represents the average surface temperature of the corresponding bodies, the Mylar is assumed to be in good enough thermal contact with the graphite and have negligible heat capacity and finally, the relaxation time for the graphite was also neglected because of its relative high thermal conductivity.

Concerning the significance of the $(K)$ matrix it is worthwhile mentioning from the beginning that its elements - i.e. the heat transfer coefficients $K_{i}(i=1,2,3)$ - do not coincide with those in reports of Domen et al. [5], who wrote the system (1) without radiative heat loss terms. Moreover, the heat transfer coefficients $K_{i}(i=1,2,3)$ defined in this paper also differ from those introduced by Janssens et al. [6]. In their model, Janssens et al. supposed that besides the heat transfer between the core and jacket there is also a direct heat transfer from core to shield - wrote the system (1), without radiative heat loss terms, in the form

$$
\left\{\begin{array}{l}
C_{1} \dot{T}_{1}=P_{1}-K_{1}\left(T_{1}-T_{2}\right)-K_{0}\left(T_{1}-T_{3}\right), \\
C_{2} \dot{T}_{2}=P_{2}+K_{1}\left(T_{1}-T_{2}\right)-K_{2}\left(T_{2}-T_{3}\right), \\
C_{3} \dot{T}_{3}=P_{3}+K_{2}\left(T_{2}-T_{3}\right)+K_{0}\left(T_{1}-T_{3}\right)-K_{3} T_{3},
\end{array}\right.
$$

where $K_{0}=1.78 \times 10^{-5} \mathrm{~W} / \mathrm{K}$ was introduced on the ground of discrepancies between theoretical and experimental heat loss corrections [16] and it represents "the direct" heat transfer coefficient $(\mathrm{W} / \mathrm{K})$ between the core and shield).

To better understand this statement let us take a deep sight on the physical significance of the heat transfer coefficients.

From a pure theoretical point of view, when a graphite calorimeter is operated in the adiabatic mode no heat transfer would take place. However, in practice there is a non-zero heat transfer across the boundaries of the bodies. The causes of this heat transfer are multiple: radiative processes, heat conduction through the thermistor wires, heat conduction and convection through the residual air, heat conduction through the supports of the bodies etc. This is why the name "quasi-adiabatic" instead of "adiabatic" is used for the corresponding operating mode of a graphite calorimeter.

Let us suppose that the calorimeter is operated in the quasi-adiabatic mode. In an electrical calibration run, equal powers are dissipated in both the core and jacket: $P_{1}=P_{2} \neq 0$, but the temperature response of only the core is measured with the Wheatstone bridge circuit shown in Fig. 3.

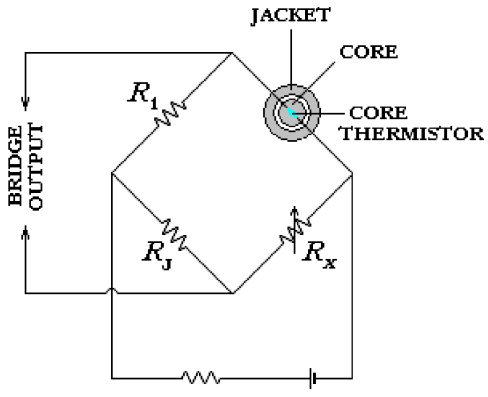

Fig. 3. The electrical circuit used for measuring the core temperature response in the quasi-adiabatic mode of operation.

Of course, some of the above enumerated causes that lead to heat losses prove to be more important and so, they must be first taken into account. For instance, as already was shown by some authors (e.g. [1]) the radiative heat loss from the core is the dominant form of heat loss for this calorimeter body in the experimental measurements, whereas all other mechanisms have considerable smaller effects.

If $P_{1}$ is the power supplied to the core during a run of duration $t_{\mathrm{h}}$, the input energy $E_{\mathrm{C}}$ (the energy supplied to the core during the run) can be expressed as

$$
E_{\mathrm{C}}=\int_{0}^{t_{\mathrm{h}}} P_{1} \mathrm{~d} t
$$

and, if the calorimeter could be operated adiabatically, then all this amount of energy would be used to increase the core temperature. In this case, with $T_{1}(0)=0$, the core temperature increase is determined by

$$
E_{\mathrm{C}}^{\text {adiabatic }}=\int_{0}^{t_{\mathrm{h}}} P_{1} \mathrm{~d} t=C_{1} T_{1}\left(t_{\mathrm{h}}\right) .
$$

Since the heating process of the core is not quite adiabatic (there is a heat loss from the core to its environment) the temperature rise of the core is smaller than $T_{1}\left(t_{\mathrm{h}}\right)$. In order to express quantitatively the fact

\footnotetext{
I "The average rate of radiative heat loss from the sample surface [has] peak losses [...] that are approximately two orders of magnitude greater than the conductive losses from the sample along the connecting thermistor leads [...] and is within $40 \%$ of the observed heat loss in the measurements" [1].
} 
that there is a non-zero heat transfer from the core to the jacket, usually a coefficient $K_{1}$ is introduced ${ }^{* *}$. If $\mathrm{d} E_{\mathrm{C}}=P_{1} \mathrm{~d} t$ is the energy delivered to the core during the infinitesimal time interval $\mathrm{d} t$ and $\mathrm{d} W$ is the infinitesimal energy loss due to the heat transfer (through all possible mechanisms) from the core to the jacket during the same time interval $(\mathrm{d} t)$, then the energy conservation law writes

$$
\mathrm{d} E_{\mathrm{C}}-\mathrm{d} W=C_{1} \mathrm{~d} T_{1}
$$

where $\mathrm{d} T_{1}$ is the corresponding infinitesimal temperature rise of the core. Obviously, $\mathrm{d} W$ is proportional to both $\mathrm{d} t$ and $T_{1}-T_{2}$ (the temperature difference between the two bodies: the core and the jacket). This proportionality can be transformed into an exact equality through a coefficient which is commonly denoted by $K_{1}$ :

$$
\mathrm{d} W \stackrel{\text { def. }}{=} K_{1}\left(T_{1}-T_{2}\right) \mathrm{d} t,
$$

and thus, the relation (16) becomes

$$
\begin{aligned}
& \mathrm{d} E_{\mathrm{C}}-K_{1}\left(T_{1}-T_{2}\right) \mathrm{d} t \\
& \quad=\left(P_{1} \mathrm{~d} t-K_{1}\left(T_{1}-T_{2}\right) \mathrm{d} t=\right) C_{1} \mathrm{~d} T_{1} .
\end{aligned}
$$

The relation (13) can be regarded as the definition-relation of the coefficient $K_{1}$. With $T_{1}(0)=0$, the integral form of relation (14) reads

$$
E_{\mathrm{C}}-K_{1} \int_{0}^{t_{\mathrm{h}}}\left(T_{1}-T_{2}\right) \mathrm{d} t=C_{1} T_{1}\left(t_{\mathrm{h}}\right)
$$

and it permits to introduce the "calculated heat loss correction factor" $F_{\mathrm{C}}[5]$ through

$$
F_{\mathrm{C}}=\frac{K_{1} \int_{0}^{t_{\mathrm{h}}}\left(T_{1}-T_{2}\right) \mathrm{d} t}{C_{1} T_{1}\left(t_{\mathrm{h}}\right)}<1,
$$

which actually represents the ratio between the lost energy $W\left(t_{\mathrm{h}}\right)$ and the energy "absorbed" by the core, $C_{1} T_{1}\left(t_{\mathrm{h}}\right)$ (which would be equal to the energy delivered to the core if there would be no heat loss, i.e. in the ideal/adiabatic case). The magnitude of this correction factor is a measure of the heat loss correction uncertainty; the smaller the calculated heat loss correction factor, the smaller the heat loss correction uncertainty is. In principle, there are at least two possible ways to reduce the size of $F_{\mathrm{C}}$. As it was already shown by Domen and Lamperti [5], one method to reduce the magnitude of this correction factor is to use the "C $+\mathrm{J}$ " mode of operating a graphite calorimeter. In this case only the core is heated $\left(P_{2}=P_{3}=0\right)$ and the core-plus-jacket temperature rise is measured by using the circuit in Fig. 4. The expression of heat loss correction factor for this case, $F_{\mathrm{C}+\mathrm{J}}$ can be theoretically determined using the first two equations in (9), in which all $e_{i}$ factors cancel, and $P_{2}=0$. The result is

\footnotetext{
** This coefficient is named "heat transfer coefficient between the core and jacket" and in SI it has the measurement units $\mathrm{W} \mathrm{K}^{-1}$. Let us note that similar coefficients are also introduced for the heat transfers between the other bodies of a graphite calorimeter.
}

$$
F_{\mathrm{C}+\mathrm{J}}=\frac{K_{2} \int_{0}^{t_{\mathrm{h}}}\left(T_{2}-T_{3}\right) \mathrm{d} t}{C_{1}\left[T_{1}\left(t_{\mathrm{h}}\right)+T_{2}\left(t_{\mathrm{h}}\right)\right]},
$$

and it can be shown that $F_{\mathrm{C}+\mathrm{J}}<F_{\mathrm{C}}$ (see Ref. [5], p. 599).

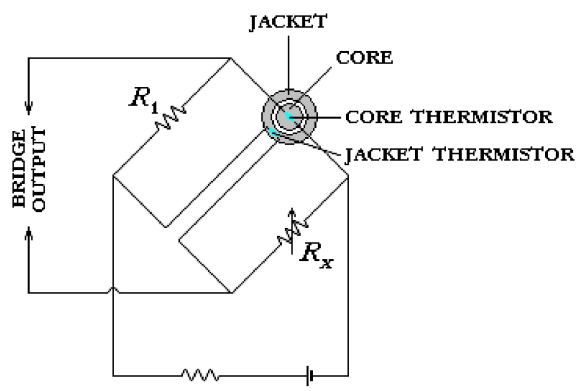

Fig. 4. The electrical circuit used for measuring the core-plus-jacket temperature response in the heat loss-compensated mode of operation.

From definition relation of $F_{\mathrm{C}}$ it results that for a given graphite calorimeter (i.e. for a fixed $C_{1}$ ) another way to decrease the correction factor $F_{\mathrm{C}}$ is to "reduce" the magnitude of the heat transfer coefficient $K_{1}$. This can be done because - from the way in which the heat transfer coefficients were introduced/defined - they "implicitly contain" the contributions from all possible heat loss mechanisms. If, for instance, all the terms that correspond to all these possible heat loss channels could be known — which is the best case - then all heat transfer coefficients must "disappear", being replaced by the sum of all these (presumable known) terms

$$
\begin{aligned}
& K_{i}=K_{i}^{\text {body surf.(rad.) }}+K_{i}^{\text {wires(cond.+rad.) }} \\
& \quad+K_{i}^{\text {resid.air(cond.) }}+K_{i}^{\text {supports(cond.) }}, \quad i=1,2,3 .
\end{aligned}
$$

Of course, in principle, for all these terms simple analytical expressions can be written down and corresponding numerical estimates can be found; this is a relatively easy task. But to find exact theoretical expressions for all these terms is a quite difficult task, because for doing this, the real spatio-temporal temperature distribution for all three bodies must be known (do not forget that during a calibration run the bodies are heated non-uniformly). Generally speaking, any known such term reduces ${ }^{\dagger \dagger}$ the size of the corresponding heat transfer coefficient by a certain amount.

$\dagger \dagger$ This method is a structural one because it takes into account the intimate "structure" of the heat transfer coefficients. Of course, for a given calorimeter the absolute/total value of any heat transfer coefficient remains in fact the same, but using this method any such transfer coefficient can be divided in one known part (containing all analytically exact-determinable heat losses) and a second "unknown" part (which contains all contributions to the total heat loss that cannot be expressed in a definite analytical form). 
There are - at least - two experimental methods to find the heat transfer coefficients [16]. One of them which is most frequently considered - uses the equilibrium temperature differences between the bodies, reached upon constant power dissipation in one or more heaters. Thus, for $K_{1}$ this method gives the following analytical expression:

$$
K_{1}=\frac{P_{1}}{T_{1}(\infty)-T_{2}(\infty)}
$$

where $T_{1}(\infty)$ and $T_{2}(\infty)$ can be experimentally determined.

Now, if only the radiative heat loss channel is considered (to be known in an analytical form), from the first equation in (6) the new "reduced" heat transfer coefficient $K_{1}^{*}$ can be defined as follows:

$$
\begin{aligned}
K_{1}^{*} & \stackrel{\text { def. }}{=} \frac{P_{1}}{T_{1}(\infty)-T_{2}(\infty)} \\
- & \frac{e_{1}\left\{\left[T_{\mathrm{M}}+T_{1}(\infty)\right]^{4}-\left[T_{\mathrm{M}}+T_{2}(\infty)\right]^{4}\right\}}{T_{1}(\infty)-T_{2}(\infty)} \\
& \stackrel{\text { not. }}{=} K_{1}-K_{1}^{\mathrm{rad}}<K_{1}
\end{aligned}
$$

and the corresponding "reduced" heat loss correction factor is defined by

$$
\begin{aligned}
F_{\mathrm{C}}^{*} & \equiv \frac{K_{1}^{*} \int_{0}^{t_{\mathrm{h}}}\left(T_{1}-T_{2}\right) \mathrm{d} t}{C_{1} T_{1}\left(t_{\mathrm{h}}\right)}=F_{\mathrm{C}} \\
- & \frac{e_{1} \int_{0}^{t_{\mathrm{h}}}\left[\left(T_{\mathrm{M}}+T_{1}\right)^{4}-\left(T_{\mathrm{M}}+T_{2}\right)^{4}\right] \mathrm{d} t}{C_{1} T_{1}\left(t_{\mathrm{h}}\right)} \\
& \stackrel{\text { not. }}{=} F_{\mathrm{C}}-F_{\mathrm{C}}^{\mathrm{rad}}<F_{\mathrm{C}},
\end{aligned}
$$

where $F_{\mathrm{C}}$ is "the old" calculated heat loss correction factor (given by relation (16)).

Analogously, the new "reduced" heat transfer coefficient $K_{2}^{*}$ can be written in terms of $K_{2}$ as follows:

$$
\begin{aligned}
K_{2}^{*} & \stackrel{\text { def. }}{=} \frac{P_{1}}{T_{2}(\infty)-T_{3}(\infty)} \\
& -\frac{e_{2}\left\{\left[T_{\mathrm{M}}+T_{2}(\infty)\right]^{4}-\left[T_{\mathrm{M}}+T_{3}(\infty)\right]^{4}\right\}}{T_{2}(\infty)-T_{3}(\infty)} \\
& =K_{2}-\frac{e_{2}\left\{\left[T_{\mathrm{M}}+T_{2}(\infty)\right]^{4}-\left[T_{\mathrm{M}}+T_{3}(\infty)\right]^{4}\right\}}{T_{2}(\infty)-T_{3}(\infty)} \\
& \stackrel{\text { not. }}{=} K_{2}-K_{2}^{\mathrm{rad}}<K_{2},
\end{aligned}
$$

and, for the "C $+\mathrm{J}$ " mode of operation,

$$
\begin{gathered}
F_{\mathrm{C}+\mathrm{J}}^{*} \equiv \frac{K_{2}^{*} \int_{0}^{t_{\mathrm{h}}}\left(T_{2}-T_{3}\right) \mathrm{d} t}{C_{1}\left[T_{1}\left(t_{\mathrm{h}}\right)+T_{2}\left(t_{\mathrm{h}}\right)\right]}=F_{\mathrm{C}+\mathrm{J}} \\
-\frac{e_{2} \int_{0}^{t_{\mathrm{h}}}\left[\left(T_{\mathrm{M}}+T_{2}\right)^{4}-\left(T_{\mathrm{M}}+T_{3}\right)^{4}\right] \mathrm{d} t}{C_{1}\left[T_{1}\left(t_{\mathrm{h}}\right)+T_{2}\left(t_{\mathrm{h}}\right)\right]} \\
\stackrel{\text { not. }}{=} F_{\mathrm{C}+\mathrm{J}}-F_{\mathrm{C}+\mathrm{J}}^{\mathrm{rad}}<F_{\mathrm{C}+\mathrm{J}}<F_{\mathrm{C}},
\end{gathered}
$$

where $F_{\mathrm{C}+\mathrm{J}}$ is that given by relation (17).

Besides, $K_{3}$ and $K_{3}^{*}$ are connected by the following relation:

$$
K_{3}^{*} \stackrel{\text { def. }}{=} \frac{P_{1}}{T_{3}(\infty)}-\frac{e_{3}\left\{\left[T_{\mathrm{M}}+T_{3}(\infty)\right]^{4}-T_{\mathrm{M}}^{4}\right\}}{T_{3}(\infty)}
$$

$$
\begin{aligned}
& =K_{3}-\frac{e_{3}\left\{\left[T_{\mathrm{M}}+T_{3}(\infty)\right]^{4}-T_{\mathrm{M}}^{4}\right\}}{T_{3}(\infty)} \\
& \stackrel{\text { not. }}{=} K_{3}-K_{3}^{\mathrm{rad}}<K_{3} .
\end{aligned}
$$

Because the small temperature rise of the shield (when the calorimeter is operated in the heat loss-compensated mode) can lead to a very inaccurate measurement, is better to determine $K_{3}$ by other means. There are at least two possibilities: (i) by analytical means, using the Stefan-Boltzmann law (as it was calculated by Domen and Lamperti [5]); (ii) using an alternative definition relation, $K_{3}=P_{3} / T_{3}$, with $P_{1}=P_{2}=0, P_{3}>0$ (as it was determined by Paulsen et al. [17]). In this last case the new "reduced" heat transfer coefficient $K_{3}^{*}$ can be defined as:

$$
\begin{aligned}
K_{3}^{*} & \stackrel{\text { def. }}{=} \frac{P_{3}}{T_{3}(\infty)}-\frac{e_{3}\left\{\left[T_{\mathrm{M}}+T_{3}(\infty)\right]^{4}-T_{\mathrm{M}}^{4}\right\}}{T_{3}(\infty)} \\
& =K_{3}-\frac{e_{3}\left\{\left[T_{\mathrm{M}}+T_{3}(\infty)\right]^{4}-T_{\mathrm{M}}^{4}\right\}}{T_{3}(\infty)} \\
& \stackrel{\text { not. }}{=} K_{3}-K_{3}^{\mathrm{rad}}<K_{3} .
\end{aligned}
$$

Having in view the above considerations, the equation system (6) must be rewritten as follows:

$$
\left\{\begin{array}{l}
C_{1} \dot{T}_{1}=P_{1}-K_{1}^{*}\left(T_{1}-T_{2}\right) \\
\quad-e_{1}\left[\left(T_{\mathrm{M}}+T_{1}\right)^{4}-\left(T_{\mathrm{M}}+T_{2}\right)^{4}\right] \\
C_{2} \dot{T}_{2}=P_{2}+K_{1}^{*}\left(T_{1}-T_{2}\right) \\
\quad-K_{2}^{*}\left(T_{2}-T_{3}\right)+e_{1}\left[\left(T_{\mathrm{M}}+T_{1}\right)^{4}-\left(T_{\mathrm{M}}+T_{2}\right)^{4}\right] \\
\quad-e_{2}\left[\left(T_{\mathrm{M}}+T_{2}\right)^{4}-\left(T_{\mathrm{M}}+T_{3}\right)^{4}\right] \\
C_{3} \dot{T}_{3}=P_{3}+K_{2}^{*}\left(T_{2}-T_{3}\right)-K_{3}^{*} T_{3} \\
\quad+e_{2}\left[\left(T_{\mathrm{M}}+T_{2}\right)^{4}-\left(T_{\mathrm{M}}+T_{3}\right)^{4}\right] \\
\quad-e_{3}\left[\left(T_{\mathrm{M}}+T_{3}\right)^{4}-T_{\mathrm{M}}^{4}\right]
\end{array}\right.
$$

where the new reduced heat transfer coefficients $K_{i}^{*}(i=$ $1,2,3)$ are defined by relations (19), (21) and (23), (24). Moreover, the presence of a supplementary coefficient $K_{0}$ in the model proves to be unnecessary ${ }^{\ddagger \ddagger}$.

\section{Results and discussion}

For a three-body Domen-type graphite calorimeter described by the characteristics given in Table, the numerical solution of system (25) is graphically presented in Figs. 5 to 11 for two cases: (a) $t_{\mathrm{h}}=100 \mathrm{~s}$ of heating followed by $t_{\mathrm{p}}=600 \mathrm{~s}$ of relaxation/post-heating time (Figs. 5 to 9 ) and (b) $t_{\mathrm{h}}=1000 \mathrm{~s}$ of heating followed by $2000 \mathrm{~s}$ of relaxation (Figs. 10 and 11).

$\ddagger \ddagger$ If the heat loss from the core through its supports and wires are taken into account the introduction of $K_{0}$ has no concrete physical support/meaning and thus becomes what in fact it is: an "artificial" way to solve the problem of discrepancies between the theory and experiment. 
To put into evidence the effect of the RHLC on each calorimeter body, in both cases the solution is given (i) without considering the radiative heat loss correction (black curves) and (ii) considering the radiative heat loss correction (red curves). Because the curve corresponding to the temperature rise of the shield is too close to the time axis, each of four "double" curves in Fig. 5 are presented separately in Figs. 6, 7, 8 and 9. Because of the same reason, this was also done for Fig. 10, but only for the double curve corresponding to the shield (that is presented separately in Fig. 11). In all the figures the superscript " $c$ " indicates that the case when heat is applied only to the core $\left(P_{1} \neq 0, P_{2}=P_{3}=0\right)$ was considered.

The numerical values of the main quantities involved in the model.

\begin{tabular}{|c|c|c|}
\hline Quantity & Symbol & Value \\
\hline heat capacity of the core & $C_{1}$ & $1.081 \mathrm{~J} \mathrm{~K}^{-1}$ \\
\hline heat capacity of the jacket & $C_{2}$ & $1.081 \mathrm{~J} \mathrm{~K}^{-1}$ \\
\hline heat capacity of the shield & $C_{3}$ & $92 \mathrm{~J} \mathrm{~K}^{-1}$ \\
\hline HTC $^{*}$ between the core and jacket & $K_{1}$ & $0.785 \times 10^{-3} \mathrm{~W} \mathrm{~K}^{-1}$ \\
\hline HTC between the jacket and shield & $K_{2}$ & $1.109 \times 10^{-3} \mathrm{~W} \mathrm{~K}^{-1}$ \\
\hline HTC between the shield and medium & $K_{3}$ & $5.2 \times 10^{-3} \mathrm{~W} \mathrm{~K}^{-1}$ \\
\hline HTC between the core and jacket in the RHLC model & $K_{1}^{*}$ & $0.539 \times 10^{-3} \mathrm{~W} \mathrm{~K}^{-1}$ \\
\hline HTC between the jacket and shield in the RHLC model & $K_{2}^{*}$ & $0.744 \times 10^{-3} \mathrm{~W} \mathrm{~K}^{-1}$ \\
\hline HTC between the shield and medium in the RHLC model & $K_{3}^{*}$ & $3.973 \times 10^{-3} \mathrm{~W} \mathrm{~K}^{-1}$ \\
\hline aluminized Mylar emissivity & $\varepsilon_{\mathrm{My}}$ & 0.044 \\
\hline graphite emissivity & $\varepsilon_{\mathrm{g}}$ & 0.82 \\
\hline RHLC coefficient between the core and jacket & $e_{1}$ & $0.232 \times 10^{-11} \mathrm{~W} \mathrm{~K}^{-4}$ \\
\hline RHLC coefficient between the jacket and shield & $e_{2}$ & $0.344 \times 10^{-11} \mathrm{~W} \mathrm{~K}^{-4}$ \\
\hline RHLC coefficient between the shield and medium & $e_{3}$ & $1.157 \times 10^{-11} \mathrm{~W} \mathrm{~K}^{-4}$ \\
\hline area of the core surface & $A_{\mathrm{c}}$ & $8.011 \times 10^{-4} \mathrm{~m}^{2}$ \\
\hline area of the interior surface of the jacket & $A_{\mathrm{j}}^{\mathrm{int}}$ & $9.5 \times 10^{-4} \mathrm{~m}^{2}$ \\
\hline area of the exterior surface of the jacket & $A_{\mathrm{j}}^{\mathrm{ext}}$ & $11.91 \times 10^{-4} \mathrm{~m}^{2}$ \\
\hline area of the interior surface of the shield & $A_{\mathrm{sh}}^{\mathrm{int}}$ & $14.06 \times 10^{-4} \mathrm{~m}^{2}$ \\
\hline area of the exterior surface of the shield & $A_{\mathrm{sh}}^{\mathrm{ext}}$ & $86.26 \times 10^{-4} \mathrm{~m}^{2}$ \\
\hline area of the interior surface of the medium & $A_{\mathrm{m}}^{\mathrm{int}}$ & $95.98 \times 10^{-4} \mathrm{~m}^{2}$ \\
\hline constant temperature of the medium & $T_{\mathrm{M}}$ & $298.15 \mathrm{~K}$ \\
\hline heating time & $t_{\mathrm{h}}$ & $100 \mathrm{~s}(1000 \mathrm{~s})$ \\
\hline post heating time & $t_{\mathrm{p}}$ & $600 \mathrm{~s}(2000 \mathrm{~s})$ \\
\hline constant power applied to the core & $P_{1}$ & $9.8 \times 10^{-5} \mathrm{~W}$ \\
\hline residual air pressure & $p$ & $5.57 \times 10^{-3} \mathrm{~Pa}$ \\
\hline
\end{tabular}

${ }^{*} \mathrm{HTC}=$ heat transfer coefficient.

Figure 6 shows that in the case (a) the core temperature rises to a maximum and decreases after power is turned off. The jacket and shield temperatures rise more slowly, since these components are not heated directly $\left(P_{2}=P_{3}=0\right)$, and continue to rise monotonously after power is turned off (see Figs. 7 and 8). For the case (b) the shape of the corresponding curves is quasi the same like in the case (a), with one exception: that regarding the jacket. When the core is heated for $1000 \mathrm{~s}$, the jacket temperature rises slowly to a maximum which does not correspond to the end of the heating time (like in the core case). It continues to rise for some time after power is turned off, then it reaches a maximum and after this it begins to decrease (see Fig. 10).

As Figs. 5, 6 and 10 show, in both (a) and (b) cases the effect of considering the RHLC for the core is a diminution of temperature (the red curves are below the corresponding black ones), and this feature lasts for the entire process (heating and post-heating periods).

On the contrary, the same effect on the jacket and shield is an increase of temperature (the red curves are situated above the corresponding black ones), and again a particular behaviour is exhibited by the jacket in the case (b) - long heating time. As Fig. 10 shows, in this 


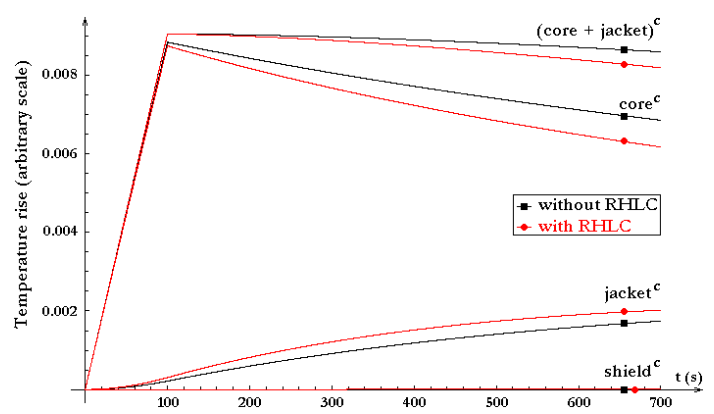

Fig. 5. Three-body calorimeter model with and without radiative heat loss correction (RHLC) for $t_{\mathrm{h}}=100 \mathrm{~s}$ of heating.

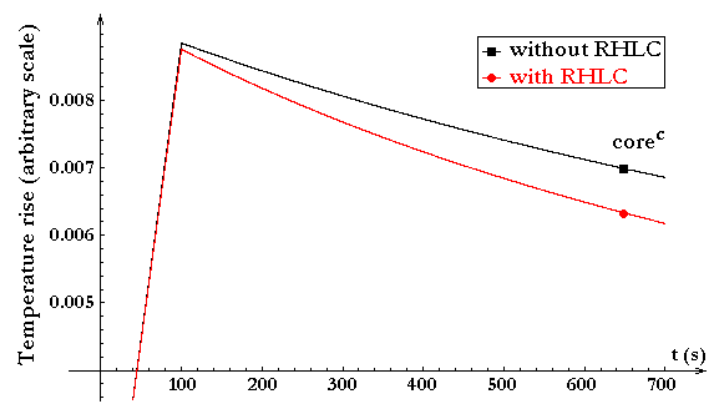

Fig. 6. Relative core's temperature-time behaviour of the three-body calorimeter for $t_{\mathrm{h}}=100 \mathrm{~s}$ of heating.

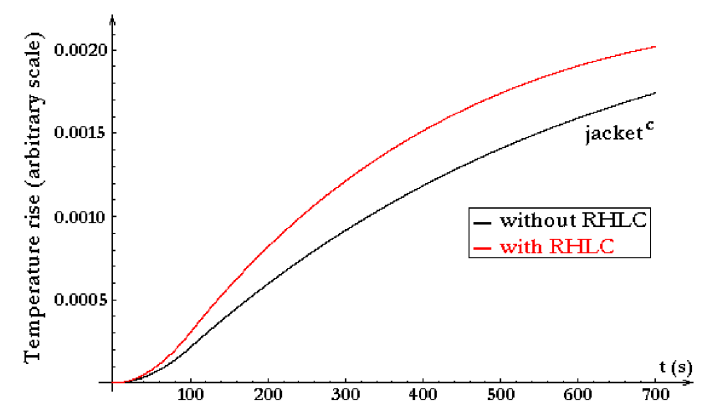

Fig. 7. Relative jacket's temperature-time behaviour of the three-body calorimeter for $t_{\mathrm{h}}=100 \mathrm{~s}$ of heating.

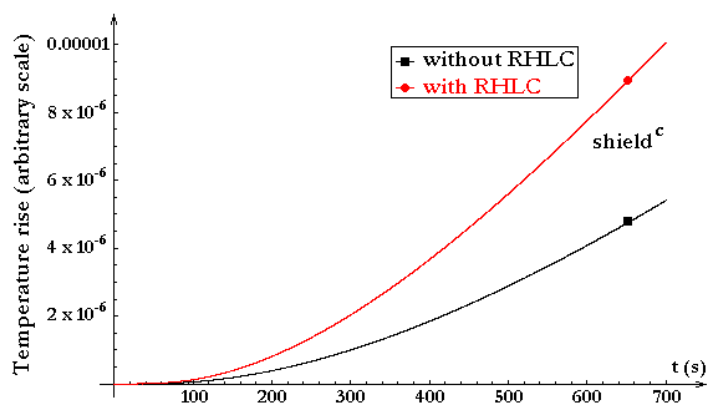

Fig. 8. Relative shield's temperature-time behaviour of the three-body calorimeter for $t_{\mathrm{h}}=100 \mathrm{~s}$ of heating.

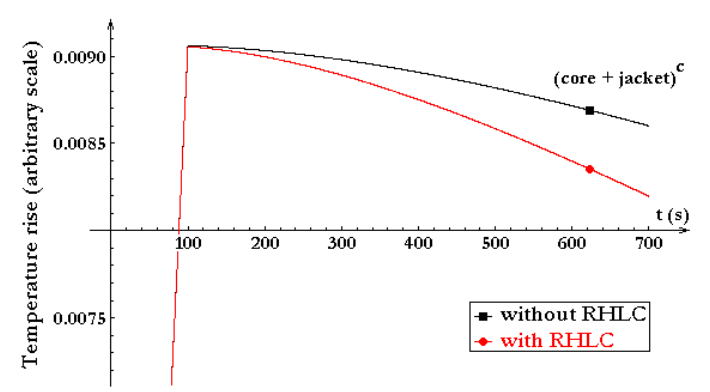

Fig. 9. Relative (core + jacket)'s temperature-time behaviour of the three-body calorimeter for $t_{\mathrm{h}}=100 \mathrm{~s}$ of heating.

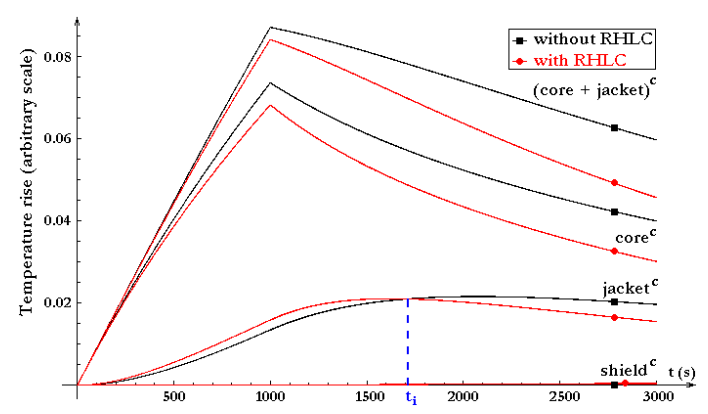

Fig. 10. Three-body calorimeter model with and without RHLC for $t_{\mathrm{h}}=1000 \mathrm{~s}$ of heating.

particular case there is an intersection point of the red and black jacket curves, where the relative position of the two curves changes. For both short and long heating times the core is a thermal radiator (it always radiates thermal energy towards its surroundings, i.e., the jacket). Apart for the case (b) regarding only the jacket, the shield and jacket are receivers for this kind of energy. The radiated thermal energy balance is positive for the jacket and shield (this means that these bodies receive more thermal energy than they emit), and it is always negative for the core.

However, if the heating time is long enough (like in the case (b)) the intermediary body (i.e. the jacket) changes

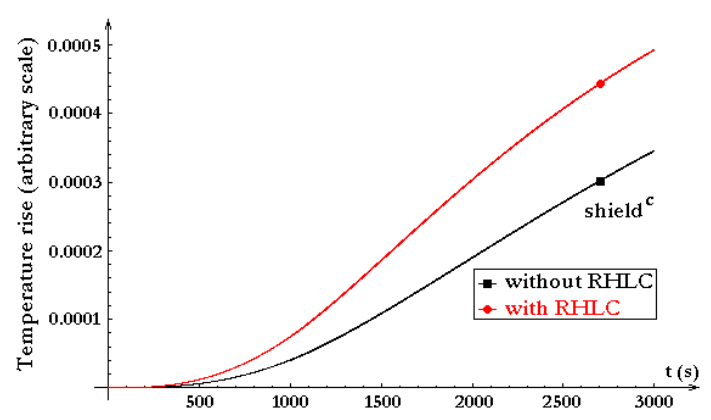

Fig. 11. Relative shield's temperature-time behaviour of the three-body calorimeter for $t_{\mathrm{h}}=1000 \mathrm{~s}$ of heating. 
its role one time during the entire process. Indeed, as Fig. 10 shows, in the first part of the process (up to the time $t_{i}$, when the two corresponding jacket curves intersect each other) the jacket is globally a receiver (it receives more radiative heat from the core than it emits towards the shield), while in the second part of the process it becomes a radiator (i.e. it emits more thermal energy towards the shield than it receives from the core).

This behaviour is normal because the jacket plays the role of a "buffer" for the process of radiative heat change between the core and shield: first, the jacket is heating since it receives more energy from the core than it emits towards the shield, but while the core is cooling, the jacket begins to emit the received energy towards the shield. In other words, when the core is hot enough it can play the role of a radiator for both surrounding bodies (the jacket and shield) because it can sustain the heating of both the bodies, but when it cannot maintain this situation (i.e. when it loses enough energy ${ }^{\S \S}$ ), the jacket takes this role in its turn but only for the shield (as it is normal).

During the initial part of the heating process, due to the small differences between the numerical values of temperature rises corresponding to the two cases (with and without RHLC), the red curves overlap on some portions of the black ones, especially for the core (see Figs. 5, 6, 9 and 10). Because of this it was preferred to represent both separately and all together the corresponding differences, $\Delta T_{i} \equiv T_{i}^{\text {without RHLC }}-T_{i}^{\text {with RHLC }}$, as functions of time $(i=1,2,3,4$ stand for core, jacket, shield and (core + jacket), respectively). The amount of these differences and their relative magnitudes emerges with a more clarity from Figs. 12 to 15 and Fig. 16, respectively. Besides, the corresponding $\Delta T_{i}(i=\overline{1,4})$ differences for $t_{\mathrm{h}}=1000 \mathrm{~s}$ of heating were graphically represented in Fig. 17, only for a comparison with dependences depicted in Fig. 16.

From Figs. 12 to 15 it results that for $t_{\mathrm{h}}=100 \mathrm{~s}$ of core heating the maximum absolute values of the differ-

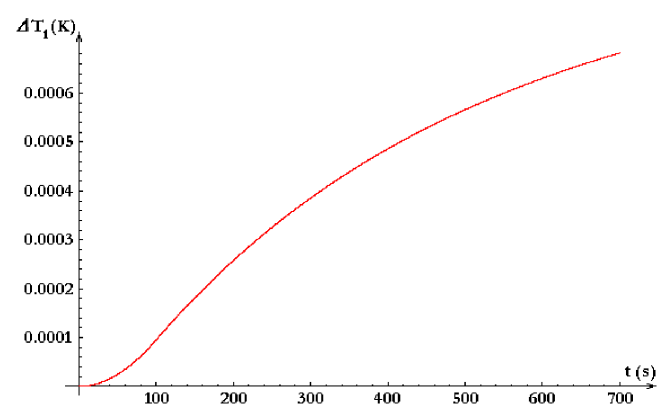

Fig. 12. Temporal dependence of $\Delta T_{\mathrm{c}}$ for $100 \mathrm{~s}$ of heating.

$\S \S$ This "enough energy" is determined by the intersection point of the red and black jacket curves in Fig. 10.

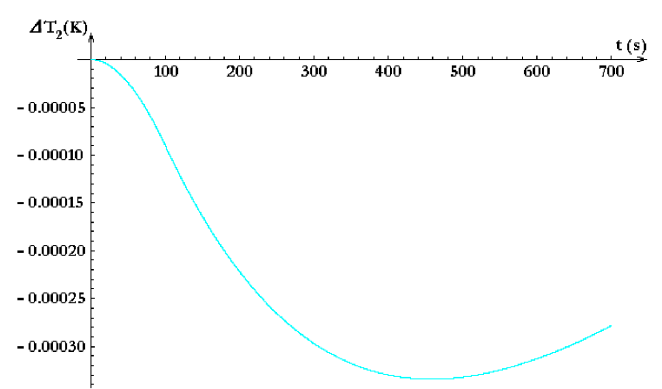

Fig. 13. Temporal dependence of $\Delta T_{\mathrm{j}}$ for $100 \mathrm{~s}$ of heating.

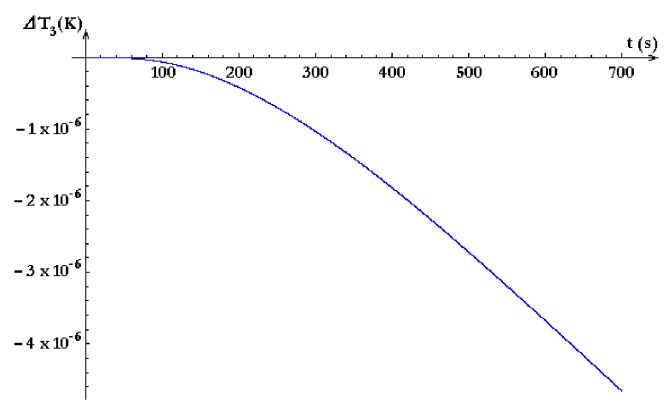

Fig. 14. Temporal dependence of $\Delta T_{\mathrm{sh}}$ for $100 \mathrm{~s}$ of heating.

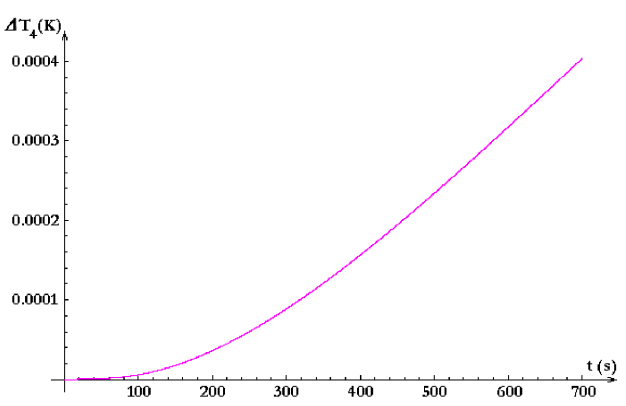

Fig. 15. Temporal dependence of $\Delta T_{\mathrm{c}+\mathrm{j}}$ for $100 \mathrm{~s}$ of heating.

ences between the two cases (with and without considering RHLC) are as follows:

- $0.68 \mathrm{mK}$ for the core - and it is obtained at the end of post heating time;

- $0.33 \mathrm{mK}$ for the jacket - and it is obtained after about $460 \mathrm{~s}$ from the initial moment;

- $4.66 \mu \mathrm{K}$ for the shield - and corresponds to the end of post heating time;

- $0.4 \mathrm{mK}$ for the core plus jacket - and corresponds to the end of post heating time. 


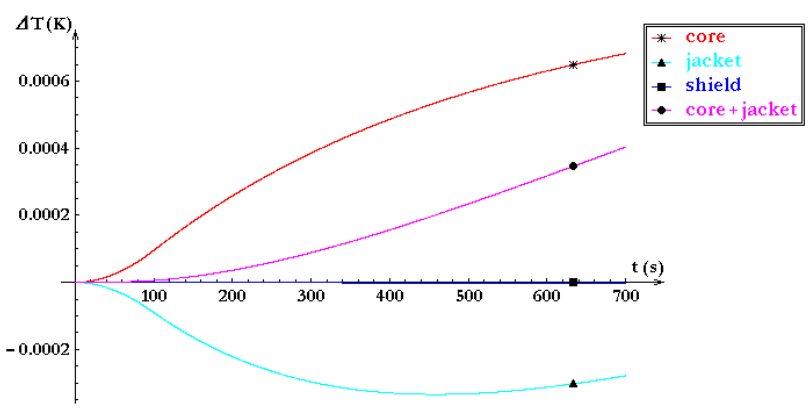

Fig. 16. Time dependences of $\Delta T_{i}(i=\overline{1,4})$ for both heating and post heating time $(700 \mathrm{~s}$ - the entire process).

The total energy lost by radiation from the core surface, $\left.W_{\mathrm{C}}^{\mathrm{rad}}\right|_{t \in\left[0, t_{\mathrm{h}}\right]}$, during the heating period $t \in\left[0, t_{\mathrm{h}}\right]$ is given by

$$
\begin{aligned}
& \left.W_{\mathrm{C}}^{\mathrm{rad}}\right|_{t \in\left[0, t_{\mathrm{h}}\right]} \\
& =e_{1} \int_{0}^{t_{\mathrm{h}}}\left\{\left[T_{\mathrm{M}}+T_{1}(t)\right]^{4}-\left[T_{\mathrm{M}}+T_{2}(t)\right]^{4}\right\} \mathrm{d} t \\
& =\frac{\sigma A_{\mathrm{c}}}{\frac{1}{\varepsilon_{\mathrm{g}}}+\frac{A_{\mathrm{c}}}{A_{\mathrm{j}}^{\text {int }}}\left(\frac{1}{\varepsilon_{\mathrm{My}}}-1\right)} \\
& \times \int_{0}^{t_{\mathrm{h}}}\left\{\left[T_{\mathrm{M}}+T_{1}(t)\right]^{4}-\left[T_{\mathrm{M}}+T_{2}(t)\right]^{4}\right\} \mathrm{d} t,
\end{aligned}
$$

where the relation $(7 \mathrm{a})$ was used.

The temperature functions $T_{1}(t)$ (for the core) and $T_{2}(t)$ (for the jacket) are the solutions of equation system (25) and they are graphically represented in Figs. 18 and 19, respectively. Because an explicit analytical form for these functions cannot be found, an integration of only numerically available solutions for $T_{1}(t)$ and $T_{2}(t)$ must be done. As Figs. 18 and 19 show, the functions $T_{1}(t)$ and $T_{2}(t)$ have a smooth enough variation on their definition intervals, and so, using an appropriate interpolation method, each of them can be precisely approximated by one polynomial only over the entire variation range. One of the best - and at the same time the easy - way to approximate a function which is given through points by a polynomial of an arbitrary order is to use the "FindFit" built-in-function in Mathematica 5.0 software. In the linear case, this function finds a globally optimal fit, whereas in the nonlinear case it generally finds a locally optimal fit. "FindFit" finds a least-squares type-fit and accepts many useful options (e.g.: PrecisionGoal, WorkingPrecision, AccuracyGoal, MaxIterations etc.) to improve the precision, accuracy and speed of calculations.

Because the time variation of the core temperature for $t \in[0,100]$ is quasi-linear, a third-order polynomial was judged to be good enough to approximate with sufficient accuracy this function. For the jacket temperature a fourth-order polynomial was chosen, since the time variation of this function is bigger than that corresponding to the core (see Figs. 18 and 19). Making the calculations by setting the WorkingPrecision to Machine-

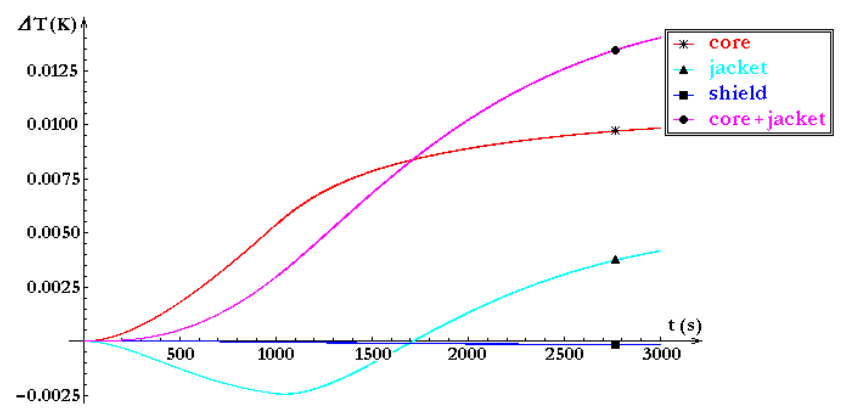

Fig. 17. Time dependences of $\Delta T_{i}(i=\overline{1,4})$ for both heating $\left(t_{\mathrm{h}}=1000 \mathrm{~s}\right)$ and post heating time.

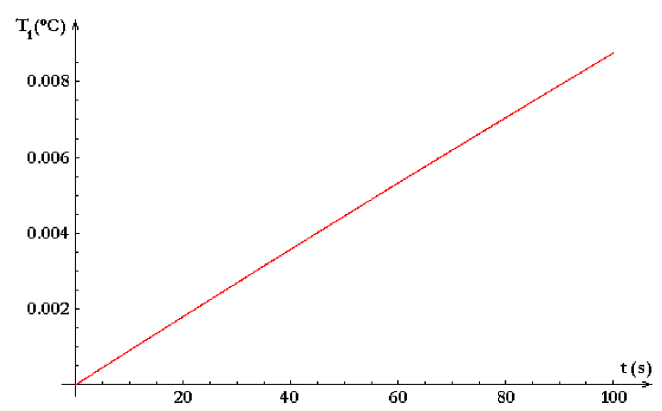

Fig. 18. Time dependence of the core temperature for $t_{\mathrm{h}}=100 \mathrm{~s}$ of heating.

-Precision $\left(53 \log _{10} 2 \approx 16\right)$ for the two functions the following approximations were found:

$$
\begin{aligned}
& T_{1}^{\mathrm{h}}(t) \equiv T_{\text {core }}^{\text {heating time }}(t) \approx c_{0 \mathrm{~h}}+c_{1 \mathrm{~h}} t+c_{2 \mathrm{~h}} t^{2}+c_{3 \mathrm{~h}} t^{3}, \\
& T_{2}^{\mathrm{h}}(t) \equiv T_{\text {jacket }}^{\text {heating time }}(t) \\
& \quad \approx j_{0 \mathrm{~h}}+j_{1 \mathrm{~h}} t+j_{2 \mathrm{~h}} t^{2}+j_{3 \mathrm{~h}} t^{3}+j_{4 \mathrm{~h}} t^{4}
\end{aligned}
$$

with

$$
\begin{gathered}
c_{0 \mathrm{~h}}=9.57105 \times 10^{-9}, \quad c_{1 \mathrm{~h}}=0.0000906548, \\
c_{2 \mathrm{~h}}=-3.28062 \times 10^{-8}, \quad c_{3 \mathrm{~h}}=1.444 \times 10^{-11}, \\
j_{0 \mathrm{~h}}=-2.06305 \times 10^{-10}, \quad j_{1 \mathrm{~h}}=6.67897 \times 10^{-11},
\end{gathered}
$$

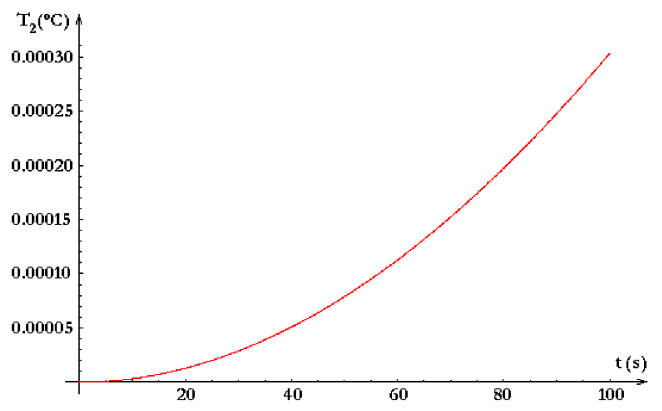

Fig. 19. Time dependence of the jacket temperature for $t_{\mathrm{h}}=100 \mathrm{~s}$ of heating. 


$$
\begin{aligned}
& j_{2 \mathrm{~h}}=3.28941 \times 10^{-8}, \quad j_{3 \mathrm{~h}}=-2.70332 \times 10^{-11}, \\
& j_{4 \mathrm{~h}}=1.33175 \times 10^{-14} .
\end{aligned}
$$

Using these data, the necessary values from Table and relation (26) as well, for the core radiative heat loss during the heating period the value was obtained

$$
\left.W_{\mathrm{C}}^{\mathrm{rad}}\right|_{t \in[0,100]}=106.35 \mu \mathrm{J},
$$

which represents $31.35 \%$ of the total core heat loss, $\left.W_{\mathrm{C}}^{\mathrm{tot}}\right|_{t \in[0,100]}=0.339 \mathrm{~mJ}$, during this time interval, and $1.1 \%$ of the total energy delivered to the core, $E_{\mathrm{c}}=$ $\int_{0}^{t_{\mathrm{h}}} P_{1} \mathrm{~d} t=9.8 \mathrm{~mJ}$. Let us note that for a configuration which is equivalent to a two-body graphite calorimeter Shipley and Duane [1] found a value of about $40 \%$ for the first percentage.

For the post heating period the time variations of the core and jacket temperatures are graphically presented in Figs. 20 and 21, respectively. Since both these time variations cannot be considered linear at all, in this case two corresponding fourth-order polynomials were chosen to approximate the functions $T_{1}(t)$ and $T_{2}(t)$ over their entire variation range, $t \in[100,700] \mathrm{s}$.

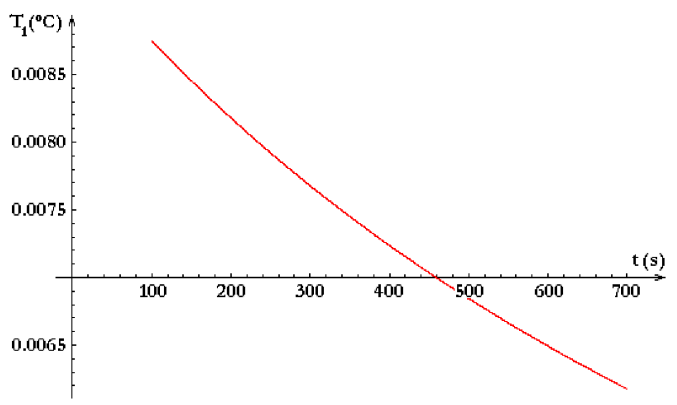

Fig. 20. Time dependence of the core temperature for post heating time, $t \in[100,700] \mathrm{s}$.

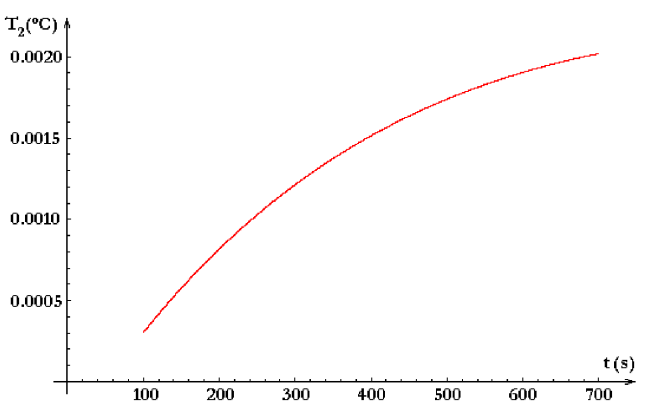

Fig. 21. Time dependence of the jacket temperature for post heating time, $t \in[100,700] \mathrm{s}$.

Following an analogous procedure like in the last case (for the heating period), these polynomials were found to be given by

$$
\begin{aligned}
& T_{1}^{\mathrm{ph}}(t) \equiv T_{\text {core }}^{\text {post heating }}(t) \\
& \quad \approx c_{0 \mathrm{p}}+c_{1 \mathrm{p}} t+c_{2 \mathrm{p}} t^{2}+c_{3 \mathrm{p}} t^{3}+c_{4 \mathrm{p}} t^{4},
\end{aligned}
$$

$$
\begin{aligned}
& T_{2}^{\mathrm{ph}}(t) \equiv T_{\text {jacket }}^{\text {post heating }}(t) \\
& \quad \approx j_{0 \mathrm{p}}+j_{1 \mathrm{p}} t+j_{2 \mathrm{p}} t^{2}+j_{3 \mathrm{p}} t^{3}+j_{4 \mathrm{p}} t^{4},
\end{aligned}
$$

where

$$
\begin{gathered}
c_{0 \mathrm{p}}=0.00940966, \quad c_{1 \mathrm{p}}=-7.0584 \times 10^{-6}, \\
c_{2 \mathrm{p}}=5.0666 \times 10^{-9}, \quad c_{3 \mathrm{p}}=-2.81651 \times 10^{-12}, \\
c_{4 \mathrm{p}}=7.97081 \times 10^{-16}, \quad j_{0 \mathrm{p}}=-0.000354319, \\
j_{1 \mathrm{p}}=7.39546 \times 10^{-6}, \quad j_{2 \mathrm{p}}=-8.6942 \times 10^{-9}, \\
j_{3 \mathrm{p}}=5.32405 \times 10^{-12}, \quad j_{4 \mathrm{p}}=-1.53395 \times 10^{-15},(31)
\end{gathered}
$$

and for the corresponding core radiative heat loss the value was found

$$
\left.W_{\mathrm{C}}^{\mathrm{rad}}\right|_{t \in[100,700]}=872.71 \mu \mathrm{J}
$$

representing $31.35 \%$ of the total core heat loss, $\left.W_{\mathrm{C}}^{\mathrm{tot}}\right|_{t \in[100,700]}=2.78 \mathrm{~mJ}$, during the post heating period.

Let us now determine the effect of the radiation heat loss correction on the absorbed dose to graphite. To this end a relation between the new calculated heat loss correction factors $F_{\mathrm{C}}^{*}$ and $F_{\mathrm{C}+\mathrm{J}}^{*}$, and the absorbed dose to graphite, $D_{\mathrm{g}}$, must be found. Having in view the definition of $D_{\mathrm{g}}$, it comes out that it is sufficient to find a relationship between the radiation energy absorbed in the core, $E_{\mathrm{C}}^{\mathrm{r}}$, and the above-mentioned two factors. This can be done if radiation runs in the $\mathrm{C}$-mode are compared with calibration runs in the $\mathrm{C}+\mathrm{J}$-mode; it results a convenient formula for $E_{\mathrm{C}}^{\mathrm{r}}$ which does not directly contain the heat capacities of the calorimeter bodies

$$
E_{\mathrm{C}}^{\mathrm{r}}=f\left(E_{\mathrm{C}+\mathrm{J}}^{\mathrm{c}}, T_{1 \mathrm{~m}}^{\mathrm{c}}, T_{1 \mathrm{~m}}^{\mathrm{r}}, T_{2 \mathrm{~m}}^{\mathrm{c}}\right) \frac{1+F_{\mathrm{C}}^{\mathrm{r}}}{1+F_{\mathrm{C}+\mathrm{J}}^{\mathrm{c}}},
$$

where $f\left(E_{\mathrm{C}+\mathrm{J}}^{\mathrm{c}}, T_{1 \mathrm{~m}}^{\mathrm{c}}, T_{1 \mathrm{~m}}^{\mathrm{r}}, T_{2 \mathrm{~m}}^{\mathrm{c}}\right)$ is a factor function which depends only on the measured calibration energy, $E_{\mathrm{C}+\mathrm{J}}^{\mathrm{c}}$, and measured temperature rises $T_{1 \mathrm{~m}}^{\mathrm{c}}, T_{1 \mathrm{~m}}^{\mathrm{r}}$ and $T_{2 \mathrm{~m}}^{\mathrm{c}}$, while $F_{\mathrm{C}}^{\mathrm{r}}$ and $F_{\mathrm{C}+\mathrm{J}}^{\mathrm{c}}$ are the calculated heat loss correction factors (for a radiation run $\boldsymbol{\top}^{\boldsymbol{\pi}}$ in the $\mathrm{C}$-mode and for an electrical calibration run in the $\mathrm{C}+\mathrm{J}$-mode, respectively). Thus, taking into account the (20) and (22) relations, it results that the searched relationship can be written as follows:

$$
D_{\mathrm{g}}=A \frac{1+\left(F_{\mathrm{C}}^{\mathrm{r}}\right)^{*}+\left(F_{\mathrm{C}}^{\mathrm{r}}\right)^{\mathrm{rad}}}{1+\left(F_{\mathrm{C}+\mathrm{J}}^{\mathrm{c}}\right)^{*}+\left(F_{\mathrm{C}+\mathrm{J}}^{\mathrm{c}}\right)^{\mathrm{rad}}},
$$

where $A$ is a proportionality factor which depends on the function factor $f$ and the mass of the core, but is independent with respect to any calculated heat loss correction factor. As it can be seen from relation (34), the radiated heat loss influences the absorbed dose $D_{\mathrm{g}}$ through the radiative components of the calculated heat loss correction factors, $\left(F_{\mathrm{C}}^{\mathrm{r}}\right)^{\mathrm{rad}}$ and $\left(F_{\mathrm{C}+\mathrm{J}}^{\mathrm{c}}\right)^{\mathrm{rad}}$. If the absorbed dose in

ๆ The lower case superscript index " $r$ " refers to a radiation run, whereas "c" designates an electrical calibration run. 
the core graphite without considering the effect of radiative heat loss correction is defined by

$$
D_{\mathrm{g}}^{*}=A \frac{1+\left(F_{\mathrm{C}}^{\mathrm{r}}\right)^{*}}{1+\left(F_{\mathrm{C}+\mathrm{J}}^{\mathrm{c}}\right)^{*}},
$$

then a new correction factor for the expression of $D_{\mathrm{g}}$ can be introduced through the obvious definition relation

$$
k_{\mathrm{SB}} \equiv \frac{D_{\mathrm{g}}}{D_{\mathrm{g}}^{*}} .
$$

This new correction factor named correction factor for the radiative heat loss from the bodies' surfaces is defined as the ratio of the absorbed dose in the graphite core considering the effect of the radiative heat loss correction from the bodies' surfaces, to the value without considering this effect. Using (20), (22), (34), (35) and (36) relations, this new correction factor can be theoretically expressed as follows:

$$
\begin{gathered}
k_{\mathrm{SB}}^{\mathrm{t}}=\frac{1+\left(F_{\mathrm{C}+\mathrm{J}}^{\mathrm{c}}\right)^{*}}{1+\left(F_{\mathrm{C}}^{\mathrm{r}}\right)^{*}} \frac{1+\left(F_{\mathrm{C}}^{\mathrm{r}}\right)^{*}+\left(F_{\mathrm{C}}^{\mathrm{r}}\right)^{\mathrm{rad}}}{1+\left(F_{\mathrm{C}+\mathrm{J}}^{\mathrm{c}}\right)^{*}+\left(F_{\mathrm{C}+\mathrm{J}}^{\mathrm{c}}\right)^{\mathrm{rad}}}=\frac{C_{1}\left[T_{1}^{\mathrm{c}}\left(t_{\mathrm{h}}^{\mathrm{c}}\right)+T_{2}^{\mathrm{c}}\left(t_{\mathrm{h}}^{\mathrm{c}}\right)\right]+\left(K_{2}^{\mathrm{c}}\right)^{*} \int_{0}^{t_{\mathrm{h}}^{\mathrm{c}}}\left(T_{2}^{\mathrm{c}}-T_{3}^{\mathrm{c}}\right) \mathrm{d} t}{C_{1} T_{1}^{\mathrm{r}}\left(t_{\mathrm{h}}^{\mathrm{r}}\right)+\left(K_{1}^{\mathrm{r}}\right)^{*} \int_{0}^{t_{\mathrm{h}}^{\mathrm{r}}}\left(T_{1}^{\mathrm{r}}-T_{2}^{\mathrm{r}}\right) \mathrm{d} t} \\
\times \frac{C_{1} T_{1}^{\mathrm{r}}\left(t_{\mathrm{h}}^{\mathrm{r}}\right)+\left(K_{1}^{\mathrm{r}}\right)^{*} \int_{0}^{t_{\mathrm{h}}^{\mathrm{r}}}\left(T_{1}^{\mathrm{r}}-T_{2}^{\mathrm{r}}\right) \mathrm{d} t+e_{1} \int_{0}^{t_{\mathrm{h}}^{\mathrm{r}}}\left[\left(T_{\mathrm{M}}+T_{1}^{\mathrm{r}}\right)^{4}-\left(T_{\mathrm{M}}+T_{2}^{\mathrm{r}}\right)^{4}\right] \mathrm{d} t}{C_{1}\left[T_{1}^{\mathrm{c}}\left(t_{\mathrm{h}}^{\mathrm{c}}\right)+T_{2}^{\mathrm{c}}\left(t_{\mathrm{h}}^{\mathrm{c}}\right)\right]+\left(K_{2}^{\mathrm{c}}\right)^{*} \int_{0}^{t_{\mathrm{h}}^{\mathrm{c}}}\left(T_{2}^{\mathrm{c}}-T_{3}^{\mathrm{c}}\right) \mathrm{d} t+e_{2} \int_{0}^{t_{\mathrm{h}}^{\mathrm{c}}}\left[\left(T_{\mathrm{M}}+T_{2}^{\mathrm{c}}\right)^{4}-\left(T_{\mathrm{M}}+T_{3}^{\mathrm{c}}\right)^{4}\right] \mathrm{d} t},
\end{gathered}
$$

where

$$
\begin{aligned}
& \left(K_{1}^{\mathrm{r}}\right)^{*} \stackrel{\text { def. }}{=} \frac{P_{1}}{T_{1}^{\mathrm{r}}(\infty)-T_{2}^{\mathrm{r}}(\infty)} \\
& -\frac{e_{1}\left\{\left[T_{\mathrm{M}}+T_{1}^{\mathrm{r}}(\infty)\right]^{4}-\left[T_{\mathrm{M}}+T_{2}^{\mathrm{r}}(\infty)\right]^{4}\right\}}{T_{1}^{\mathrm{r}}(\infty)-T_{2}^{\mathrm{r}}(\infty)} \\
& =K_{1}^{\mathrm{r}}-\left(K_{1}^{\mathrm{r}}\right)^{\mathrm{rad}}<K_{1}^{\mathrm{r}}, \\
& \left(K_{2}^{\mathrm{c}}\right)^{*} \equiv K_{2}^{*}, \quad K_{1}^{\mathrm{r}} \equiv \frac{P_{1}}{T_{1}^{\mathrm{r}}(\infty)-T_{2}^{\mathrm{r}}(\infty)} \\
& =\frac{P_{1}}{T_{1}(\infty)-T_{2}(\infty)}=K_{1}, \quad t_{\mathrm{h}}^{\mathrm{c}}=100 \mathrm{~s}, \\
& t_{\mathrm{h}}^{\mathrm{r}}=240 \mathrm{~s}, \quad T_{i}^{\mathrm{c}}, \quad(i=\overline{1,3})
\end{aligned}
$$

are the solutions of the equation system $(25)$ with $P_{1} \neq 0$ and $P_{2}=P_{3}=0, T_{i}^{\mathrm{r}},(i=\overline{1,3})$ are the solutions of the same equation system but with $P_{1} / C_{1}=P_{2} / C_{2}=P_{3} / C_{3}$ and

$$
\left\{\begin{array}{l}
T_{1}^{\mathrm{r}}(\infty)=P_{1} / K_{1}+T_{2}^{\mathrm{r}}(\infty), \\
T_{2}^{\mathrm{r}}(\infty)=\left(1+K_{3} / K_{2}\right) T_{3}^{\mathrm{r}}(\infty)-\left(P_{1} / K_{2}\right)\left(C_{3} / C_{1}\right), \\
T_{3}^{\mathrm{r}}(\infty)=\left(P_{1} / K_{3}\right)\left(2+C_{3} / C_{1}\right) .
\end{array}\right.
$$

Making all necessary substitutions in (37) and integrating for the new correction factor $k_{\mathrm{SB}}$ it results in a theoretical value of

$$
k_{\mathrm{SB}}^{\mathrm{t}}=0.999623 \approx 0.9996 .
$$

If $k_{\mathrm{SB}}$ is determined directly from the amount of radiative heat lost from the core surface during the heating period $\left(t_{\mathrm{h}}^{\mathrm{c}}=100 \mathrm{~s}\right)$ of an electrical calibration run, by taking into consideration the view-emissivity factor [18], then it results that

$$
k_{\mathrm{SB}}=\frac{9800 \mu \mathrm{J}-3.447 \mu \mathrm{J}}{9800 \mu \mathrm{J}}=0.999648 \approx 0.9996,
$$

i.e., $k_{\mathrm{SB}}$ has approximately the same value as $k_{\mathrm{SB}}^{\mathrm{t}}$ (the difference between the two values is very small, being of
$0.0025 \%$ ). The value of $k_{\mathrm{SB}}$ is less important than the correction for vacuum gaps, correction for density difference between the phantom and calorimeter, correction for depth measurement, correction for the calorimeter front slab and correction for impurities, it is more important than each of the following corrections: correction for air attenuation, correction for the front kapton foil, correction for difference of thermal transfer between electrical calibration and irradiation and correction for the poly (methyl methacrylate) (PMMA) back and radial thickness, and it has the same order of magnitude as the correction for absorbed dose gradient in the core and correction for source-reference point distance [19].

It is important to notice that, if $k_{\mathrm{SB}}$ is calculated using this alternative, the amount of radiative heat loss from the core surface during a radiation run can be neglected because it is very small: $\left.\left(W_{\mathrm{C}}^{\mathrm{r}}\right)^{\mathrm{rad}}\right|_{t \in[0,240] \mathrm{s}}=$ $1.58 \times 10^{-7} \mathrm{~J}$. The very small difference between the two values obtained for the new introduced correction factor (the theoretically calculated value, $k_{\mathrm{SB}}^{\mathrm{t}}=0.999623$, and the value determined directly from the amount of radiative heat loss, $\left.k_{\mathrm{SB}}=0.999648\right)$ is due to the non-zero effect of RHLC on the factors $\left(F_{\mathrm{C}}^{\mathrm{r}}\right)^{*}$ and $\left(F_{\mathrm{C}+\mathrm{J}}^{\mathrm{c}}\right)^{*}$ in relation (35) through the temperatures $T_{1}(t), T_{2}(t)$ and $T_{3}(t)$ - on the one hand, and through the new heat transfer coefficients $K_{1}^{*}$ and $K_{2}^{*}$ - on the other hand (see the relations (20) and (22)). In conclusion, if the model based on differential equation system (25) is adopted for describing a 3-body Domen-type graphite calorimeter, then the absorbed dose to graphite must be corrected by the factor $k_{\mathrm{SB}} \approx 0.9996$ (or, in percent, $0.04 \%$ ).

\section{Conclusions}

Taking into account the radiative heat loss correction, a new refined ordinary differential equation model for 
describing the global behaviour of a three-body Domen-type graphite calorimeter was proposed and analyzed. This model is more precise than the old one and, thus, can lead to results closer to reality (i.e., to experimental measurements). Besides, in the case of long-enough period of core heating (tens of minutes), it was revealed that the jacket plays the role of a sui-generis buffer for the radiated heat, and thus, the usefulness of the third calorimeter body (the shield) was theoretically settled when building a graphite calorimeter, which, for the first time was introduced by Domen [5].

The new "reduced" heat transfer coefficients that were defined in this improved model, $K_{i}^{*}=K_{i}-K_{i}^{\mathrm{rad}},(i=$ $1,2,3)$, allowed to define new (calculated) heat loss correction factors, $F_{\mathrm{C}}^{*}<F_{\mathrm{C}}$ and $F_{\mathrm{C}+\mathrm{J}}^{*}<F_{\mathrm{C}+\mathrm{J}}$, which are smaller than the old ones for both the radiation and calibration runs; consequently, the heat loss correction uncertainty decreases.

The model permits the calculation of the radiated heat from the calorimeter bodies' surfaces for both heating and post heating periods, which could be used in determining the corresponding new correction factor for the absorbed dose to graphite. This new correction factor named correction factor for the radiative heat loss from the bodies' surfaces was defined as the ratio of the absorbed dose in the core graphite considering the effect of the radiative heat loss correction from the bodies' surfaces, to the value without considering this effect. Two quasi-equal values (determined by two different means) were calculated for this new correction factor: a pure theoretical one, $k_{\mathrm{SB}}^{\mathrm{t}}=0.999623 \approx 0.9996$, and one inferred from the effective amount of heat lost through radiation from the core surface, $k_{\mathrm{SB}}=0.999648 \approx 0.9996$.

The amount of heat lost through radiation was calculated only for the core surface for both heating and post heating periods and it was found that in both cases it represents $31.35 \%$ from the corresponding total core heat losses.

\section{Acknowledgments}

Dr. D. Radu would like to acknowledge the financial support of the ICTP, Trieste, Italy, through the TRIL (Training and Research in Italian Laboratories) Programme which made possible this research.

\section{References}

[1] D.R. Shipley, S. Duane, Heat Loss Mechanisms in a Measurement of Specific Heat Capacity of Graphite, CIRA (EXT) 003, January 1996.

[2] E.H. Kennard, Kinetic Theory of Gases, McGraw-Hill, New York 1938.
[3] S. Dushman, in: Vacuum Technique, Wiley, New York 1949 , p. 47.

[4] B. Todd, J.B. Young, J. Power Sources 110, 186 (2002).

[5] S.R. Domen, P.J. Lamperti, J. Res. NBS - A. Phys. Chem. 78A, 596 (1974).

[6] A. Janssens, E. Cottens, A. Paulsen, A. Poffijn, Metrologia 22, 265 (1986).

[7] S. Picard, D.T. Burns, P. Roger, Prog. Rep. CCRI(I)/05-30, 22 April 2005.

[8] S. Picard, D.T. Burns, P. Roger, Measurement of the Specific Heat Capacity of Graphite, Rapport BIPM$-2006 / 01,2006$.

[9] J. Daures, A. Ostrowsky, Phys. Med. Biol. 50, 4035 (2005).

[10] J. Witzani, K.E. Duftschmid, Ch. Strachotinsky, A. Leitner, Metrologia 20, 73 (1984).

[11] S.R. Domen, in: The Dosimetry of Ionizing Radiation, Vol. II, Eds. K.R. Kase, B.E. Bjarngard, F.H. Attix, Academic Press, Inc., Harcourt Brace Jovanovich, Publishers, Orlando 1987, p. 258.

[12] W.H. McAdams, Heat Transmission, 2nd ed., McGraw-Hill, New York 1942, p. 45.

[13] S.R. Domen, Radiat. Phys. Chem. 37, 199 (1991).

[14] D.R. Lide, R.C. Weast, CRC Handbook of Chemistry and Physics, 71st ed., CRC Press 1990-1991.

[15] FAR Assoc. web-page: Non Contact Temperature Measurement for Process Industries, Advanced Materials and Research; Practical Multi-Wavelength Pyrometry, http://www.pyrometry.com/ emissivity.php, Graphite emissivity as a function of temperature.

[16] E. Cottens, A. Janssens, G. Eggermont, R. Jacobs, in: Proc. Int. Symp. Biomedical Dosimetry, Paris 1980, IAEA-SM-249/32, 1981, p. 189.

[17] A. Paulsen, R. Widera, H. Nerb, L. van Rhee, The CBNM Graphite Absorbed-Dose Calorimeter, $\mathrm{GE} / \mathrm{R} / \mathrm{VG} / 52 / 86$, private communication, 1986.

[18] D. Radu, A.S. Guerra, C. Ionita, I. Astefanoaei, Metrologia 47, 179 (2010).

[19] J. Daures, A. Ostrowsky, P. Gross, J.P. Jeannot, J. Gouriou, in: Proc. NPL Workshop on Recent Advances in Calorimetric Absorbed Dose Standards, CIRM 42, 1999, Eds. A.J. Williams, K.E. Rosser, National Physical Laboratory, Teddington (UK) 2000, p. 15 . 